\title{
Transplantation of bone marrow mesenchymal stromal cells attenuates liver fibrosis in mice by regulating macrophage subtypes
}

Xiao-Yu Luo ${ }^{1}$, Xiang-Jun Meng ${ }^{1 *}$, Da-Chun Cao ${ }^{1 *}$, Wei Wang ${ }^{1,2}$, Kun Zhou' ${ }^{1}$ Lei Li', Mei Guo ${ }^{1}$ and Ping Wang ${ }^{3}$

\begin{abstract}
Background: Liver fibrosis is a key phase that will progress to further injuries such as liver cirrhosis or carcinoma. This study aimed to investigate whether transplantation of bone marrow mesenchymal stromal cells (BM-MSCs) can attenuate liver fibrosis in mice and the underlying mechanisms based on the regulation of macrophage subtypes.

Methods: A liver fibrosis model was induced by intraperitoneal (i.p.) injection of CCl4 twice per week for 70 days, and BM-MSCs were intravenously transplanted twice on the 60th and 70th days. Immunohistology and gene expression of liver fibrosis and macrophage subtypes were analyzed. Mouse RAW264.7 cells and JS1 cells (hepatic stellate cell strain) were also used to explore the underlying mechanisms of the effects of BM-MSCs on liver fibrosis.

Results: After transplantation of BM-MSCs, $\mathrm{F} 4 / 80^{+} \mathrm{CD} 206^{+}$-activated M2 macrophages and matrix metalloproteinase 13 (MMP 13) expression were significantly increased while $\mathrm{F} 4 / 80^{+} \mathrm{iNOS}^{+}$-activated $\mathrm{M} 1$ macrophages were inhibited in liver tissue. Gene expression of IL-10 was elevated while IL12b, IFN- $\gamma$, TNF- $a$, and IL-6 gene expression were decreased. TGF- $\beta 1$ and collagen-1 secretions were reduced while caspase-3 was increased in JS1 cells treated with BM-MSC-conditioned media. BM-MSCs effectively suppressed the expression of a-SMA, Sirius red, and collagen-1 in the liver, which are positively correlated with fibrosis and induced by $\mathrm{CCl} 4$ injection.

Conclusions: Taken together, we have provided the first demonstration that BM-MSC transplantation can promote the activation of M2 macrophages expressing MMP13 and inhibition of M1 macrophages to further inhibit hepatic stellate cells (HSCs), which play synergistic roles in attenuating liver fibrosis.
\end{abstract}

Keywords: Mesenchymal stromal cell, Macrophage, Liver fibrosis, Matrix metalloproteinase 13, Hepatic stellate cell

\section{Background}

Epidemiological analysis has revealed that liver fibrosis/ cirrhosis is a severe health problem worldwide that accounts for substantial morbidity and mortality. Of the 1.4 million liver disease deaths each year, 55\% are attributed to liver cirrhosis [1-4]. Liver fibrosis is a key period in the development of almost any liver disease that involves gradual destruction and will progress to liver cirrhosis or

\footnotetext{
* Correspondence: mxj2m9h@163.com; caodachun@yeah.net ${ }^{1}$ Department of Gastroenterology, Shanghai Ninth People's Hospital, Shanghai Jiao Tong University School of Medicine, Center for Specialty Strategy Research of Shanghai Jiao Tong University China Hospital Development Institute, No 639, Zhizaoju Road, Huangpu District, Shanghai 200011, China

Full list of author information is available at the end of the article
}

carcinoma. There are few effective treatments for curing liver fibrosis/cirrhosis and carcinoma, and liver transplantation remains the only option, which is restricted by a lack of donor organs and lifelong immunological rejection.

Mesenchymal stromal cells (MSCs) are currently attracting great attention from researchers because they are associated with fewer ethical concerns than embryonic stem cells; on the other hand, they are poor stimulators of the allogeneic $\mathrm{T}$ cell response in vitro and do not trigger a strong host inflammatory response in vivo $[5,6]$ because they only express low levels of type I HLA and do not express type II HLA and the costimulatory molecules CD40, CD80, and CD86 [5]. Recent studies have demonstrated that MSCs can be transplanted into baboons or even

(C) The Author(s). 2019 Open Access This article is distributed under the terms of the Creative Commons Attribution 4.0 International License (http://creativecommons.org/licenses/by/4.0/), which permits unrestricted use, distribution, and 
humans with beneficial effects and without immunological rejection, as well in most animals $[7,8]$.

Among various types of MSCs, bone marrow mesenchymal stromal cells (BM-MSCs) are now preferred not only due to their easy isolation and high expandability but also for their thoroughly characterized phenotypic expression, cytokine secretion, and paracrine activity [9]. Since Friedenstein et al. first described BM-MSCs [10], many properties of BM-MSCs have been reported, such as connecting different tissues, secreting various growth factors, anti-inflammation, and immunoregulation. BMMSCs have been shown to play an anti-fibrosis role in animal models and in several human clinical trials [11, 12]. There are different perspectives about the anti-fibrosis mechanisms of BM-MSCs, and the latest research indicates that BM-MSCs reduce liver fibrosis via immunosuppressive and anti-inflammatory activities, such as inhibitory efforts on natural killers (NK) cells, dendritic cells, and Th1 cell proliferation and activation of M2 macrophages and Th2 cells [13]. Due to the abundance of innate immune cells in the liver, the polarization of macrophages after BM-MSC transplantation attracted our interest.

Macrophages include different subtypes, mainly M1 and M2 macrophages, according to their different surface markers, gene expression profiles, and activated effects [14, 15]. There have been few studies of the influence of macrophage subtypes on the liver fibrosis process. Initially, studies reported that M2 macrophages stimulated the development of liver fibrosis while M1 macrophages suppressed fibrosis, but Pesce et al. subsequently demonstrated that activated M2 macrophages inhibited fibrosis $[16,17]$. Recently, a study reported that M1 macrophages accelerate the process of liver fibrosis [18]. And previous studies have shown that MMPs are essential for fibrinolysis, and MMP13 in particular, as the major interstitial collagenase in rodents, plays a crucial role in the resolution and cleavage of fibrous collagen [19-22]. However, no study has demonstrated relationships or mechanisms linking the transplantation of BM-MSCs and macrophage polarization with the expression of associated matrix metalloproteinases (MMPs) in a liver fibrosis model. Consequently, we examined the effect of the administration of BM-MSCs on liver fibrosis in mice and investigated the impact of BM-MSC transplantation on the regulation of macrophage subtypes and MMPs expression to determine the therapeutic potential of BM-MSCs in liver fibrosis.

\section{Material and methods}

\section{Animal models}

All animals received humane care, and all methods were carried out in accordance with the Guide for the Care and Use of Laboratory Animals. The experiments were approved by the Committee on the Ethics of Animal Experiments of
Shanghai Jiao Tong University. Ten-week-old male C57BL/ 6) mice weighing $25-27 \mathrm{~g}$ were housed four per cage in temperature- and light-controlled chambers. There are a variety of experimental liver fibrosis models, but the CCl4induced model appears to be the most classic and widely applied [23, 24]. In this study, liver fibrosis was induced by i.p. injection of $\mathrm{CCl} 4$ dissolved in olive oil at a volume ratio of 1:1 at a dose of $0.1 \mathrm{ml} /$ mouse twice per week for 70 days. The animals were randomized into three groups as follows: (1) normal control group $(n=10)$-treated with i.p. injection of saline twice per week for 70 days; (2) fibrosis group $(n=$ 10) - treated with i.p. injection of CCl4 twice per week for 70 days; and (3) fibrosis+MSC group $(n=12)$-treated with $\mathrm{CCl} 4$ twice per week for 70 days and treated with an injection of BM-MSCs via the tail vein at a dose of $5 \times 10^{5}$ on the 60th day and 70th day. Animals were sacrificed on the 80th day, and each liver was excised and divided into several parts for hematoxylin-eosin (HE) staining, immunohistochemical staining, immunofluorescence staining, and RNA extraction. In addition, five mice were treated with $\mathrm{CCl} 4$ followed by transplantation of GFP-positive BM-MSCs to assess the migration of the transplanted cells.

\section{Isolation, expansion, and characterization of BM-MSCs}

BM-MSCs were isolated and cultured as described in a previous study [25]. Briefly, after the donor mice were sacrificed, the cleaned bones of the tibia and femur were stored in DMEM (Gibco, Carlsbad, CA, USA) supplemented with penicillin/streptomycin on ice. The bone marrow was extracted by inserting a 27-gauge needle attached to a 10-ml syringe containing DMEM with strong flushing to remove the growth plates of the bones. The cell suspension was filtered through a $70-\mu \mathrm{m}$ filter mesh and then cultured in a $60-\mathrm{mm}$ culture dish in $1 \mathrm{ml}$ of complete medium at a density of $25 \times 10^{6} / \mathrm{ml}$. The plate was incubated at $37^{\circ} \mathrm{C}$ with $5 \% \mathrm{CO}_{2}$ in a humidified chamber. After $6 \mathrm{~h}$, nonadherent cells were removed by replacing the medium with fresh complete medium. After an additional $6 \mathrm{~h}$ of culture, the medium was replaced by $1.5 \mathrm{ml}$ of fresh complete medium. Afterwards, the medium was changed every $8 \mathrm{~h}$ for up to 3 days of initial culture. Then, adherent cells were washed with PBS, and the medium was replaced with $6 \mathrm{ml}$ of fresh medium every 3 to 4 days. From the third day, spindle-shaped cells appeared and expanded to become increasingly confluent. At the third week, highly purified BM-MSCs were obtained. And the cells from P5-7 were used for further experiments in this study.

The BM-MSCs were characterized using suitable markers by flow cytometric analysis. BM-MSCs were $\mathrm{CD} 54^{+} \mathrm{CD} 90^{+}$ $\mathrm{CD} 11^{-}$in this experiment. The FACS analysis was performed using a CyAn ADP flow cytometer (Beckman Coulter). All data were analyzed by FlowJo software (TreeStar, Inc). Differentiation experiments were performed using a mesenchymal stromal cell adipogenic differentiation kit 
and osteogenic differentiation kit (R\&D Systems Minneapolis, MN, USA) to establish the reliability of the BM-MSCs.

Histopathological and immunohistochemical examination Liver tissue samples were stored in 10\% formalin solution. Paraffin blocks were prepared as 4- $\mu \mathrm{m}$ cross sections, and $\mathrm{HE}$ staining and Sirius red staining were performed. Formalin-fixed and paraffin-embedded sections of the livers were also used in the immunohistochemical examination, and $\alpha$-smooth muscle actin $(\alpha-S M A)$ staining was applied to show the activation of HSCs (dilution 1:100; Dako Japan, Tokyo, Japan). Collagen-1 staining was conducted to show the fibrous collagen of liver fibrosis (dilution 1:100; Abcam, Cambridge, MA, USA). The fibrotic areas were observed in three sections per mouse.

\section{Immunofluorescence staining}

Liver tissue was immediately obtained when the mice were sacrificed and subsequently dehydrated in 30\% sucrose PBS solution, embedded in Tissue-Tek OCT compound (Sakura Finetek USA, Inc., Torrance, CA, USA), and snap-frozen in dry ice. Frozen sections with a thickness of $6 \mu \mathrm{m}$ were fixed in $4 \%$ paraformaldehyde, blocked with $5 \%$ goat serum, and incubated at $4{ }^{\circ} \mathrm{C}$ overnight with primary antibodies against $\mathrm{F} 4 / 80$ (marker of mouse monocytes/macrophages), iNOS (marker of mouse M1 macrophages), CD206 (marker of mouse M2 macrophages), and MMP13. All of the above antibodies (Abcam, Cambridge, MA, USA) were diluted $1: 100$. The frozen sections were incubated with appropriate fluorescein-conjugated secondary antibodies for $2 \mathrm{~h}$ at room temperature. The fluorescence was examined and photographed using a Lecia fluorescence microscope.
RNA preparation and quantitative reverse transcriptase polymerase chain reaction

Total RNA was extracted from frozen liver tissue using Isogen (Nippon Gene, Tokyo, Japan). Each 800-ng RNA sample was reverse-transcribed to cDNA using oligo (dT) primers and SuperScript reverse transcriptase (Invitrogen, Life Technologies Japan) according to the manufacturer's protocol. The target-specific primers were designed as listed in Table 1. Quantitative RT-PCR was performed using a TaqMan system on an Applied Biosystems PRISM 7700 device (ABI Japan, Co., Ltd., Tokyo, Japan) with 0.9 $\mathrm{mM}$ each primer in a final reaction volume of $25 \mu \mathrm{l}$ of Premix Ex TaqTM (Takara Bio Inc., Shiga, Japan). The PCR cycling conditions were as follows: $50{ }^{\circ} \mathrm{C}$ for $2 \mathrm{~min}, 95^{\circ} \mathrm{C}$ for $15 \mathrm{~min}$, and $50 \mathrm{cycles}$ of $95^{\circ} \mathrm{C}$ for $30 \mathrm{~s}, 60^{\circ} \mathrm{C}$ for $1 \mathrm{~min}$, and $25^{\circ} \mathrm{C}$ for $2 \mathrm{~min}$. The data were expressed as the comparative cycle threshold $\left(C_{t}\right)$ values. The normalized $C_{t}$ value of each gene was obtained by subtracting the $C_{t}$ value of $18 \mathrm{~s}$ rRNA.

\section{M1 macrophage polarization and co-culture assay}

Murine RAW264.7 cells and JS1 cells (hepatic stellate cell strain) obtained from the Cell Bank of the Chinese Academy of Sciences (Shanghai, China) were used for further experiments in this study which were from P4-5. The cells were cultured in DMEM supplemented with 10\% FBS (Gibco, USA), $100 \mathrm{U} / \mathrm{ml}$ penicillin, and $100 \mu \mathrm{g} / \mathrm{ml}$ streptomycin at $37^{\circ} \mathrm{C}$ in a humidified $5 \% \mathrm{CO}_{2}$ atmosphere. For experiments, the RAW264.7 cells (seeded at $3 \times 10^{5} / \mathrm{ml}$ ) were stimulated with $100 \mathrm{ng} / \mathrm{ml}$ LPS for $6 \mathrm{~h}$ as described previously [26] to yield M1 macrophage polarization.

JS1 cells and LPS-stimulated M1 macrophages from RAW264.7 cells were co-cultured in two chambers separated by a semipermeable membrane with a pore size of $1 \mu \mathrm{m}$ to prevent contact between the cells. JS1 cells were cultured in the upper insert of the chamber, while M1 macrophages were cultured in the lower chamber. In

Table 1 The primers used in this study

\begin{tabular}{|c|c|c|}
\hline Genes & Forward (5'-3') & Reverse $\left(5^{\prime}-3^{\prime}\right)$ \\
\hline $\mathrm{IL}-6$ & CTGCAAGTGCATCATCGTTGT & TGTCTATACCACTTCACAAGTCGGA \\
\hline TNF-a & TGTCTACTGAACTTCGGGTGAT & AACTGATGAGAGGGAGGCCAT \\
\hline $\mathrm{IFN}-\gamma$ & CAAGGCGAAAAAGGATGCA & CGGATGAGCTCATTGAATGCT \\
\hline IL-10 & GGGTGAGAAGCTGAAGACCCT & TCACCTGCTCCACTGCCTT \\
\hline TGF- $\beta 1$ & AGGTCACCCGCGTGCTAA & GCTTCCCGAATGTCTGACGTA \\
\hline a-SMA & CTGACAGAGGCACCACTGAA & CATCTCCAGAGTCCAGCACA \\
\hline $\mid L-12 b$ & CTCAGAAGCTAACCATCTCCTGG & CACAGGTGAGGTTCACTGTTTC \\
\hline MMP13 & TTATGGTCCAGGCGATGAAG & AGGCGCCAGAAGAATCTGTC \\
\hline Collagen-1 & GAGCGGAGAGTACTGGATCG & TACTCGAACGGGAATCCATC \\
\hline Collagen-4 & GGTATTCAGGGAGACCGTGG & ACCCTTGTGCACCCCTAGAT \\
\hline \multirow[t]{2}{*}{$18 \mathrm{~s}$} & ATGAGTCCACTTAA & CTITAATATACGCT \\
\hline & AATCCTTTAACGA & ATTGGAGCTGGAA \\
\hline
\end{tabular}


addition, BM-MSC-conditioned media was added to the chamber containing M1 macrophages for stimulation, and the impact of the influence of the BM-MSCs on M1 macrophages on the activation or apoptosis of JS1 cells was observed. After $48 \mathrm{~h}$ of co-culture, the upper inserts were removed, and the levels of TGF- $\beta 1$ and collagen- 1 in the supernatant of the JS1 cells were measured by ELISA (R\&D Systems Minneapolis, MN, USA). Caspase-3 was measured in lysed JS1 cells. The levels of TGF- $\beta 1$ and collagen-1 were also measured in the supernatants of M1 macrophages and JS1 cells when they were cultured alone with or without BM-MSC-conditioned media.

\section{Measurement of caspase-3 enzyme activity in JS1 cells}

Caspase- 3 enzyme activity was measured using a Caspase- 3 Activity Assay kit (Beyotime, Shanghai, China) according to the manufacturer's instructions. Briefly, JS1 cells were harvested after $48 \mathrm{~h}$ of culture alone or co-culture with LPS-stimulated M1 macrophages from RAW264.7 cells that were treated with or without BM-MCS-conditioned media. The harvested cells were then lysed in cold lysis buffer and centrifuged at $15,000 \mathrm{~g}$ for $5 \mathrm{~min}$. The supernatant was transferred to ice-cold fresh tubes for immediate assay. The assay was based on the spectrophotometric detection of chromophore p-nitroaniline ( $p$-NA) after cleavage from the labeled substrate DEVD- $p$-NA. The $p$-NA fluorescence emission was quantified at $405 \mathrm{~nm}$, and finally, the caspase- 3 activity was determined by comparison of the absorbance of $p$-NA from the treated sample with that of the control.

\section{Statistical analysis}

The results were presented as the means $\pm \mathrm{SE}$, and the data were analyzed using the statistical software package SPSS 12.0 (SPSS Inc., Chicago, IL, USA). The groups were compared by one-way ANOVA, followed by Fisher's protected least significance difference test or the Mann-Whitney $U$ test. Values of $p<0.05$ were considered to be statistically significant.

\section{Results}

\section{Characterization of BM-MSCs}

BM-MSCs were isolated and cultured following our above protocol. The BM-MSCs reached 25-35\% confluence after 7 days and $75-85 \%$ confluence after 14 days. After 21 days of culture, the essentially uniform spindle-shaped BM-MSCs reached greater than $92 \%$ confluence as assessed by phase-contrast microscopy (Fig. 1a), and more than $70 \%$ of cells had a colony-formation capacity, consistent with a previous report [25]. Furthermore, we verified the purification process and reliability of the BM-MSCs by flow cytometric analysis and differentiation experiments. $\mathrm{CD}^{1} 1^{+}$mononuclear macrophages and granulocytes gradually decreased while $\mathrm{CD} 90^{+} \mathrm{CD} 54^{+} \mathrm{CD} 11^{-} \mathrm{BM}-\mathrm{MSC}$ gradually increased from the first week to third week (Fig. 1c). The BM-MSCs exhibited little contamination by hematopoietic or other cell lineages after 3 weeks of culture (Fig. 1a, c). Differentiation experiments showed that the BM-MSCs successfully differentiated into adipocytes and osteoblasts after 3 weeks of induction, based on visualization of oil droplets in the cultured cells by positive Oil Red O staining and calciumcontaining precipitates by staining with $2 \%$ Alizarin red adjusted to a pH of 4.4 with ammonium hydroxide (Fig. 1b).

\section{BM-MSCs migrated to the injured livers and attenuated the loss of body weight and liver injury}

To assess the migration of transplanted BM-MSCs, we injected $5 \times 10^{5}$ BM-MSCs from GFP mice in each liver-injured recipient via the tail vein. Abundant GFPpositive BM-MSCs were detected in the recipient mice after transplantation (Fig. 2a). The number of GFP-pos itive cells reached a maximum between 12 and $36 \mathrm{~h}$ after transplantation.

From the beginning of the experiment until the 60th day, the average body weight was not significantly different between the fibrosis group and fibrosis+MSC group. However, after injection of BM-MSCs twice on the 60th day and 70th day, the loss of body weight slowed in the fibrosis + MSC group. At the end point (the 80th day) of the experiment, the weight decreased by $9.3 \mathrm{~g}$ on average in the fibrosis group and 7.4 $\mathrm{g}$ on average in the fibrosis+MSC group, corresponding to approximately $35 \%$ and $28.2 \%$, respectively, of their initial body weights (Fig. 2b). In addition, the injection of $\mathrm{CCl} 4$ twice per week for 70 days resulted in 13and 5.5-fold increases in serum alanine aminotransferase (ALT) and aspartate aminotransferase (AST) levels, respectively, compared with normal mice; however, transplantation of BM-MSCs effectively inhibited the increase in serum aminotransferase (Fig. 2c).

\section{Transplantation of BM-MSCs suppressed liver fibrosis} Mice that did not receive $\mathrm{CCl} 4$ injection displayed normal histology, while mice that received $\mathrm{CCl} 4$ injection twice per week for 70 days developed obvious hepatic fibrosis. By contrast, mice that received CCl4 and BM-MSC injections displayed an apparent decrease in fibrosis (Fig. 3a). Furthermore, we detected $\alpha$-SMA, Sirius red, and collagen-1 staining, which represent the extent of liver fibrosis. Consistent with the HE staining, $\alpha$-SMA staining showed numerous positive cells located around the central vein areas and infiltrated into the middle part of the lobules in the fibrosis group. However, $\alpha$-SMA-positive areas were significantly reduced in the fibrosis+MSC group (Fig. 3b). Sirius red staining showed marked perisinusoidal collagen deposition starting from the central district and extending into the hepatic lobules, which occupied $25 \%$ of the liver area in the fibrosis group, while this amount of collagen was decreased to $12 \%$ of the liver area in the fibrosis+MSC 

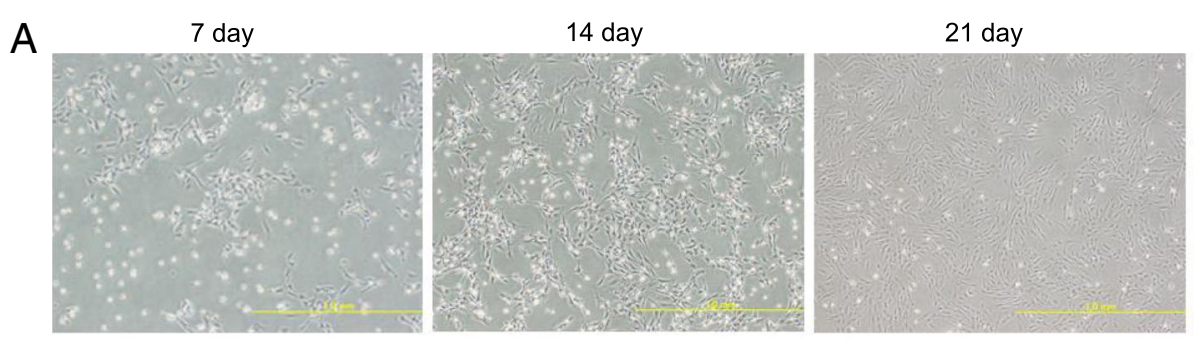

B
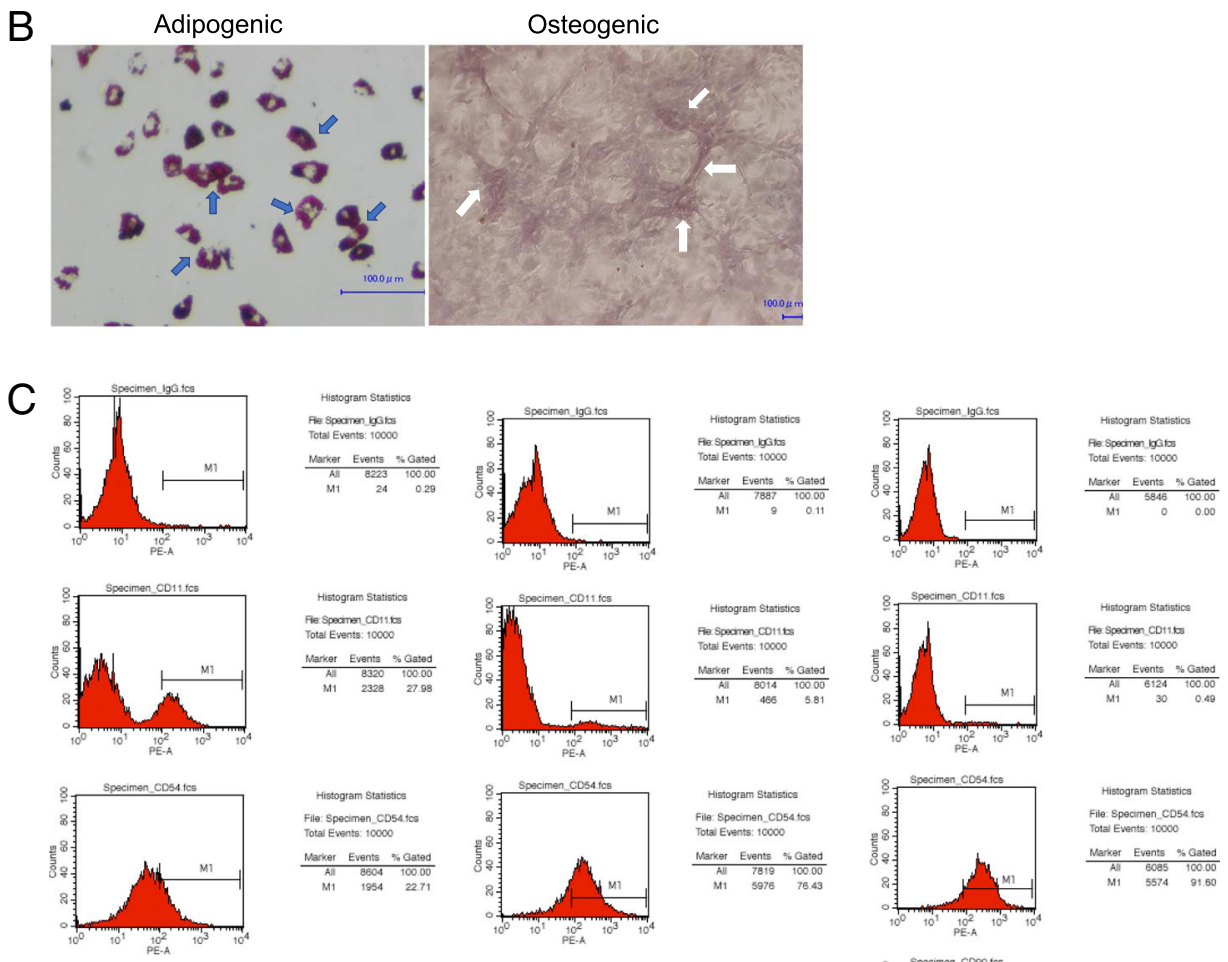

Histogram Statisicics

File: Spocimen cD54titos
Total Events: 10000

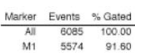
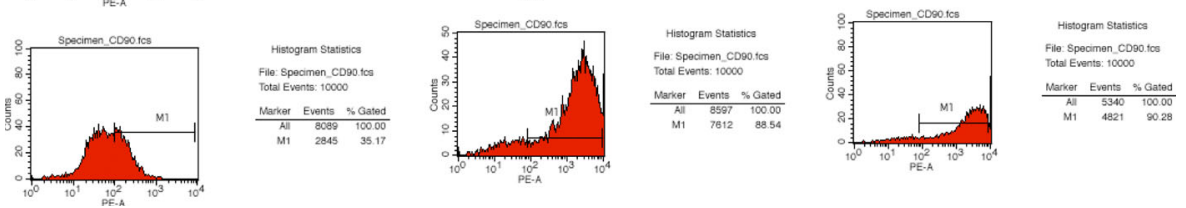

Fig. 1 Morphological, immunophenotypic, and differentiation analysis of BM-MSCs. a Morphological pictures of BM-MSCs after 7 days, 14 days, and 21 days of culture. The scale bars represent $1.0 \mathrm{~mm}$. b BM-MSCs differentiated into adipocytes (blue arrows) and osteoblasts (white arrows). The scale bars represent $100 \mu \mathrm{m}$. c Flow cytometric analysis of BM-MSCs (CD90 $\left.{ }^{+} \mathrm{CD} 54^{+} \mathrm{CD} 11^{-}\right)$in different culture time

group (Fig. 3c). The collagen-1 staining results were similar to the $\alpha$-SMA and Sirius red staining results (Fig. 3d).

We also measured fibrosis-related gene expression levels in liver tissue, including TGF- $\beta 1, \alpha$-SMA, colla gen- 1 , and collagen- 4 . TGF- $\beta 1$ was largely secreted by activated HSCs, which will accelerate the formation of liver fibrosis, and $\alpha$-SMA was expressed mainly by myofibroblasts derived from activated HSCs. The mRNA expression levels of the above four genes were low in normal mice and were obviously increased by CCl4 administration. Notably, the mRNA expression levels of these genes were dramatically decreased by BM-MSC transplantation (Fig. 3e).

\section{Transplantation of BM-MSCs increased the M2/M1 macrophage ratio}

Immunofluorescence staining of M1 and M2 macrophage markers revealed some interesting changes in macrophage subtypes. F4/80 ${ }^{+} \mathrm{iNOS}^{+}$cells represented activated M1 macrophages, while $\mathrm{F} 4 / 80^{+} \mathrm{CD} 206^{+}$cells represented activated M2 macrophages. M1 macrophages dramatically increased in fibrotic livers induced by CCl4 but decreased significantly after BM-MSC transplantation (Fig. 4b, c). However, M2 macrophages displayed an obvious decrease in the fibrosis group but a dramatic increase in the fibrosis+MSC group (Fig. 4e, f). Thus, transplantation of BM-MSCs effectively increased the M2/M1 macrophage ratio in the liver (Fig. 4g). 


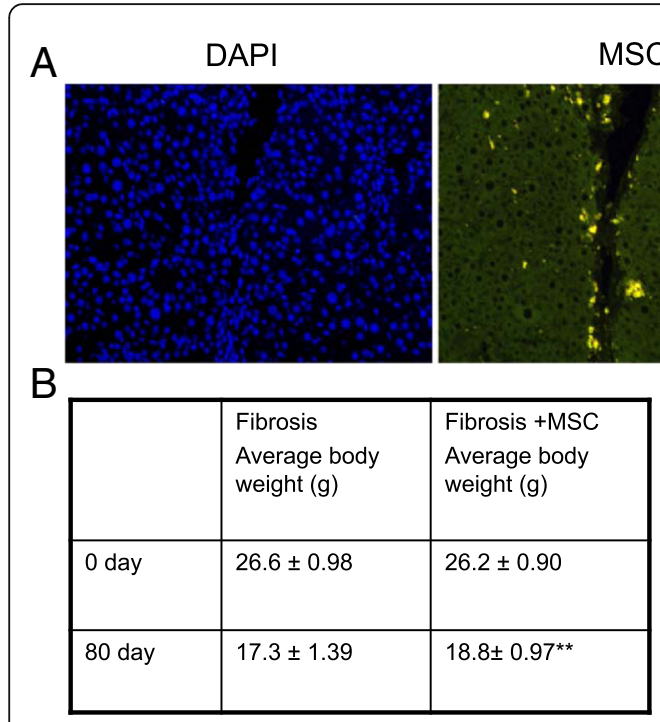

MSC
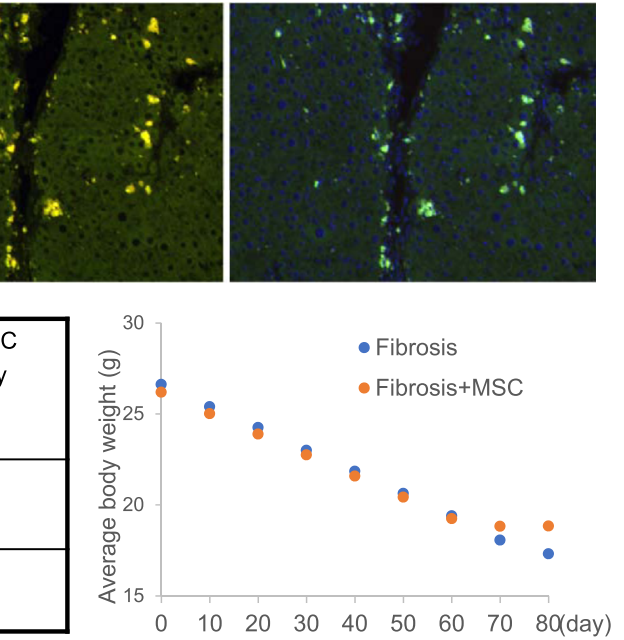

Merge

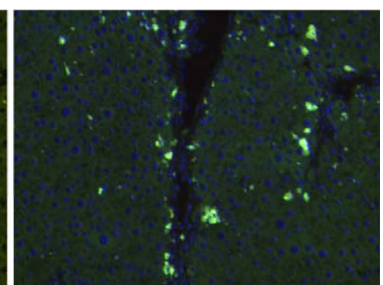

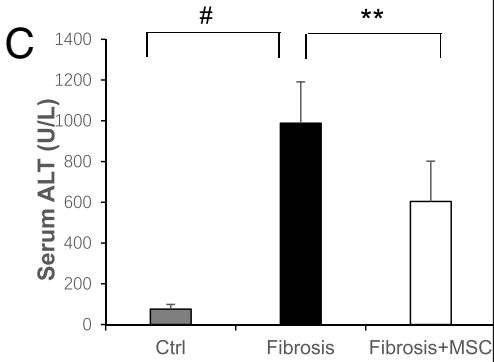

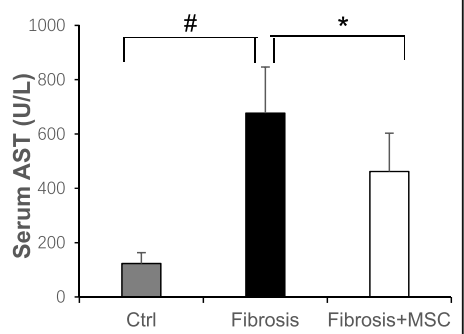

Fig. 2 Transplanted BM-MSCs migrated to the injured liver and alleviated the loss of body weight and liver injury. a Migration of GFP-positive BM-MSCs into the liver after $24 \mathrm{~h}$ ( $\times 200$ magnification). b The body weight loss in the fibrosis group and fibrosis+MSC group. c The ALT and AST levels in each group. (BM-MSCs were from P5-7, means \pm SE; ${ }^{*} p<0.01$ vs. the normal control group, ${ }^{* *} p<0.01$ vs. the fibrosis group, ${ }^{*} p<0.05$ vs. the fibrosis group)

To further confirm the effect of BM-MSCs on regulating macrophage subtypes, we detected $\mathrm{M} 1$ and $\mathrm{M} 2$ macrophage-related cytokines. IL12b is derived from M1 macrophages, and IL-10 is mainly derived from M2 macrophages [27]. We found that the mRNA level of IL12b was significantly increased in the fibrotic livers but decreased after transplantation of BM-MSCs two times. However, IL-10 mRNA showed a different change trend, with a marked increase in the fibrosis+MSC group compared with the fibrosis group (Fig. 4h). In addition, the IL-10/IL12b ratio was decreased in the fibrotic liver induced by $\mathrm{CCl} 4$ but increased by the administration of BM-MSCs (Fig. 4i). We also measured the expression of M1 macrophage-related inflammatory factors including

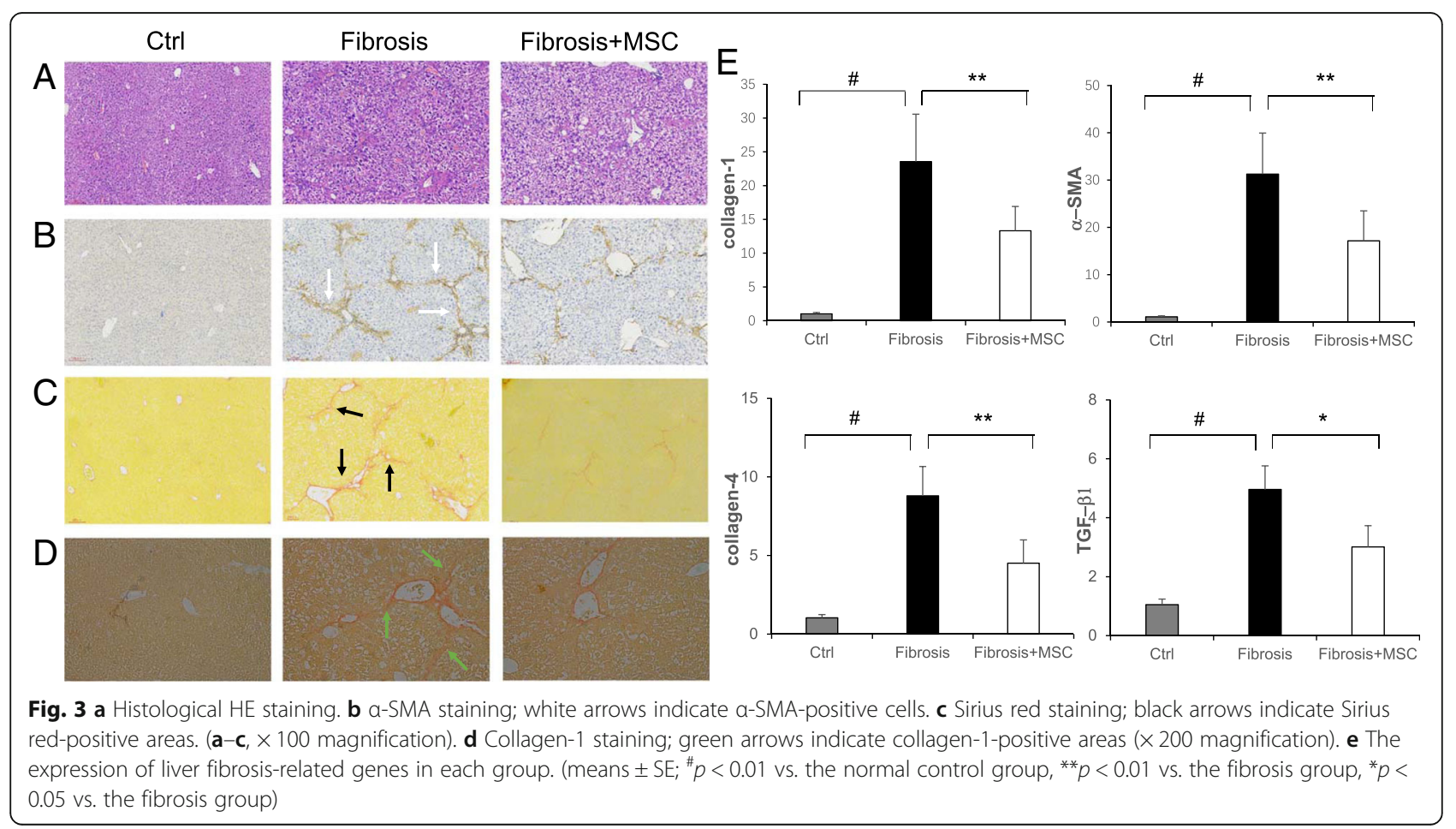




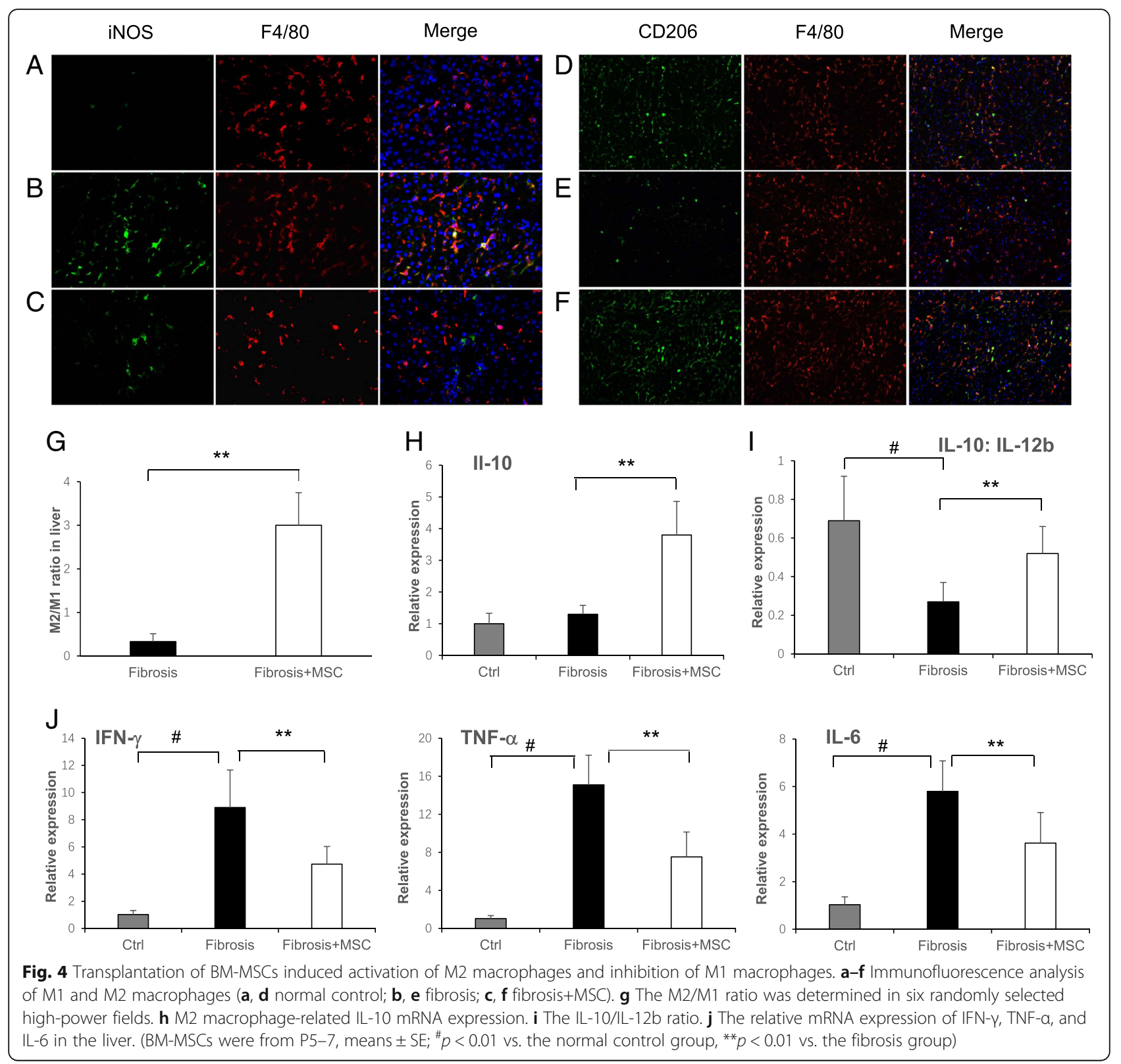

IFN- $\gamma$, TNF- $\alpha$, and IL- 6 in liver tissue, which simultaneously increased in the fibrosis group but obviously decreased in the fibrosis+MSC group (Fig. 4j).

\section{BM-MSC transplantation increased the expression of MMP13 by activated M2 macrophages}

The components of fibrous collagen are mainly type I collagen in liver fibrosis, which is largely degraded by MMP1 in humans. Rodent MMP1 has not been identified, but studies have shown that MMP13 plays an equivalent role in rodents [19]. Immunofluorescence staining of MMP13 showed that MMP13 expression was increased in the fibrosis+MSC group compared with the fibrosis group (Fig. 5a). In addition, the mRNA expression level of MMP13 was consistent with the results of immunofluorescence staining (Fig. 5b). Moreover, we performed double immunofluorescence staining of MMP13 and CD206 and found that the positive areas of MMP13 expression and CD206 expression overlapped by more than $90 \%$ (Fig. 5c). These results verified our speculation that the increased MMP13 expression was derived from activated and proliferative M2 macrophages.

\section{Effect of BM-MSC-conditioned media on HSCs via M1 macrophages}

TGF- $\beta 1$ and collagen- 1 were detected by ELISA in the supernatants of six groups: JS1, JS1+MSC, M1, M1 +MSC, JS1+M1, and JS1+M1+MSC. The results revealed 


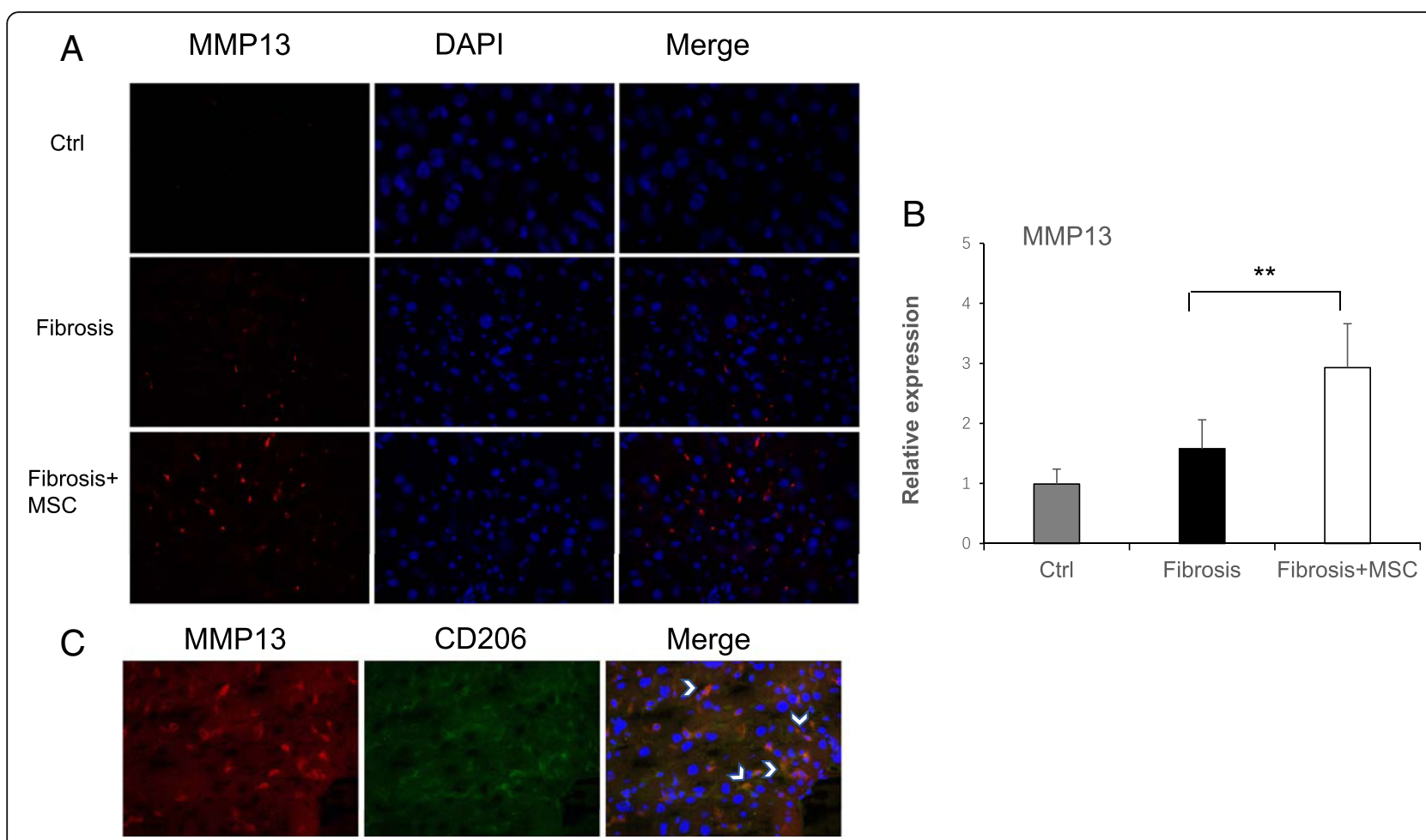

Fig. 5 a Immunofluorescence staining of MMP13 in the liver. $\mathbf{b}$ The relative mRNA expression of MMP13 in each group (means $\pm S E ;{ }^{* *} p<0.01$ vs. the fibrosis group). c Double immunofluorescence staining; the white arrowheads indicate MMP13-positive cells where CD206 was co-immunolocalized

that the concentrations of TGF- $\beta 1$ and collagen- 1 in JS1 cells cultured alone were increased by 3.1 - and 2.8 -fold, respectively, compared with M1 macrophages cultured alone. No obvious changes in concentration were detected after incubation with BM-MSC-conditioned media. However, the levels of TGF- $\beta 1$ and collagen-1 were apparently increased in the JS1+M1 co-culture group compared with JS1 cells cultured alone. Furthermore, the addition of BM-MSC-conditioned media to M1 macrophages in the co-culture group ultimately reduced the levels of secreted TGF- $\beta 1$ and collagen- 1 in JS1 cells (Fig. 6a, b). We also detected the apoptosis of JS1 cells when cultured alone or co-cultured with M1 macrophages treated with or without BM-MSC-conditioned media. The results showed that caspase-3 was decreased in the co-culture compared with JS1 cells cultured alone. In addition, caspase- 3 production in the co-culture was significantly increased after BM-MSC-conditioned media was added to the M1 macrophage culture (Fig. 6c).

\section{Discussion}

Liver fibrosis, the key phase that may eventually progress to liver cirrhosis or hepatocellular carcinoma, is a complex and kinetic process involving various cell types and cytokines that result in the activation of hepatic stellate cells and accumulation of excessive extracellular matrix (ECM). However, there are no satisfactory treatments for liver fibrosis. New therapies such as stromal cell transplantation have shown improvements in liver biochemical parameters and histological evaluation, and further research on these therapies is urgently needed.

In our study, CCl4 induced a loss of body weight and increased ALT and AST levels, although some previous studies reported that body weight increased slightly in mice receiving $\mathrm{CCl} 4$ injections for 6 to 8 weeks compared to the initial body weight. Notably, the injection of $\mathrm{CCl} 4$ induced a dramatic decrease in body weight compared with the normal control in all of those studies, similar to our data. Importantly, BM-MSC transplantation attenuated the loss of body weight and inhibited the increase in aminotransferases in our present study (Fig. 2b, c). We attributed these changes to the induction of hepatocyte injury by $\mathrm{CCl} 4$ injection and the reduced synthesis of albumin in the liver, which further induced the loss of body weight; transplantation of BM-MSCs rectified this hepatocyte injury and restored liver function to a certain extent.

As expected, $\mathrm{CCl} 4$ injection promoted obvious liver fibrosis, which was effectively attenuated by BM-MSC transplantation in our present study. Immunohistochemical staining of $\alpha$-SMA showed that $\mathrm{CCl} 4$ induced a notable increase in myofibroblasts, and this increase was reduced significantly by transplantation of BM-MSCs (Fig. 3b). $\alpha$-SMA-positive cells were used to represent myofibroblasts, which are derived from activated HSCs 


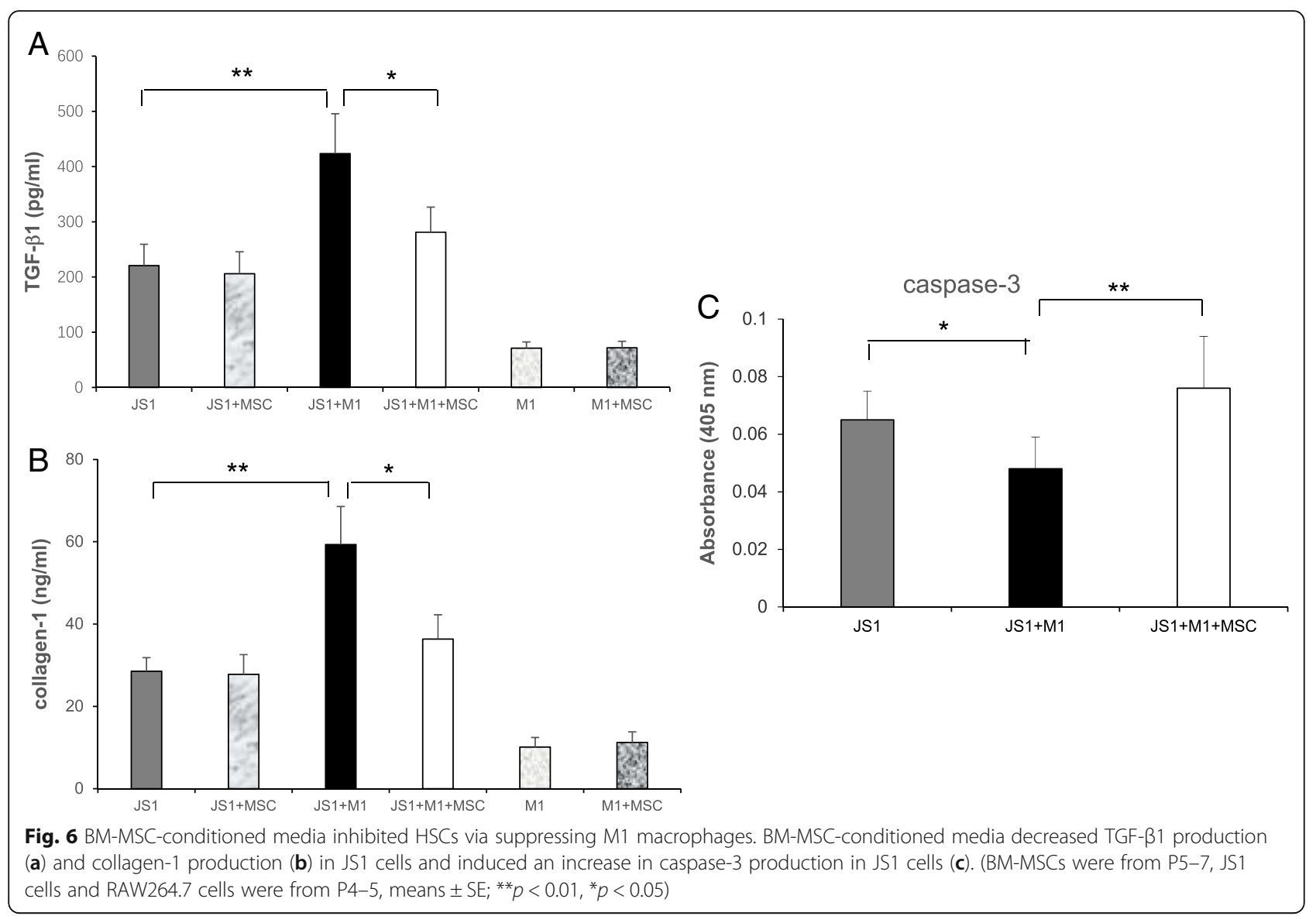

[28], and it is universally accepted that activation of HSCs plays a key role in the process of liver fibrosis [29]. The change in positive areas of Sirius red and collagen-1 staining also demonstrated the effect of BM-MSCs in reducing liver fibrosis (Fig. 3c, d). The mRNA expression levels of TGF- $\beta 1, \alpha$-SMA, collagen- 1 , and collagen- 4 further corroborated these results (Fig. 3e).

Notably, immunofluorescence staining of macrophage markers showed that $\mathrm{CCl} 4$ injection induced the proliferation of M1 macrophages, while BM-MSC transplantation induced the proliferation and activation of M2 macrophages and inhibition of M1 macrophages (Fig. 4b, c, e, f). M1 and M2 macrophage-related cytokines in liver tissue showed similar changes. Mice that received CCl4 followed by BM-MSC transplantation displayed a significant increase in IL-10 compared with mice that received CCl4 injection only, and the ratio of IL-10/IL12b was apparently increased by the administration of BM-MSCs (Fig. 4h, i). This result is also supported by a previous study that revealed that human amniotic epithelial cell transplantation induced markers of alternative macrophage activation [27].

Furthermore, our study not only demonstrated that transplantation of BM-MSCs alleviated liver fibrosis but also revealed that this alleviation effect was due to the elevated expression of MMP13 (Fig. 5a, b), which was consistent with previous studies reported MMPs especially MMP13 was the major interstitial collagenase in rodents, played a crucial role in degrading fibrous collagen [1922]. Importantly, MMP13 was expressed by activated M2 macrophages in our present study (Fig. 5c), which accounted for the positive connection between the activation of M2 macrophages and attenuation of liver fibrosis. Hence, we concluded that transplantation of BM-MSCs attenuated liver fibrosis by activating M2 macrophages, which were able to express MMP13.

In addition, we detected the expression of inflammatory cytokines including IFN- $\gamma$, TNF- $\alpha$, and IL- 6 , which simultaneously increased after $\mathrm{CCl} 4$ injection but decreased significantly after BM-MSC transplantation two times (Fig. 4j). The levels of the above inflammatory cytokines were closely associated with the change in M1 macrophages, demonstrating that these cytokines were mainly derived from M1 macrophages, consistent with results reported by Subramanian [30]. Our previous study provided apparent evidence of IFN- $\gamma$ dependence of liver fibrosis [31]. Studies have also demonstrated that TNF- $\alpha$ and IL-6 play important roles in the development of liver fibrosis [32-34]. Our observations that CCl4 injection induced elevation of IFN- $\gamma$, TNF- $\alpha$, and IL- 6 and aggravation of liver fibrosis in this study are consistent with these previous 
conclusions. Taken together, these results indicated that the fibrosis-alleviating effect of BM-MSC transplantation was accompanied by a decrease in M1 macrophages and inhibition of the above relevant inflammatory cytokines. The inhibition of the activation of M1 macrophages, suppression of inflammatory cytokines, and decrease in HSC-related fibrous collagen after BM-MSC transplantation led us to hypothesize that the transplanted BM-MSCs probably inhibited a pathway from activation of M1 macrophages to activation of HSCs that normally would trigger the differentiation of $\alpha$-SMA-positive myofibroblasts under stimulation. Hence, we further analyzed the influence of BMMSCs on HSCs via M1 macrophages in vitro. The results showed that M1 macrophages induced the activation of HSCs; however, BM-MSCs were able to ultimately suppress the activation effect and accelerate the apoptosis of HSCs (Fig. 6).

\section{Conclusions}

In conclusion, irritative factors such as $\mathrm{CCl} 4$ injection stimulate the proliferation of M1 macrophages, which further trigger HSC activation into $\alpha$-SMA-positive myofibroblasts to accelerate the development of liver fibrosis by expressing TNF- $\alpha$, IFN- $\gamma$, and IL- 6 . During the development of liver fibrosis, at least in the CCl4-induced liver fibrosis model, M2 macrophages were suppressed. However, transplantation of BM-MSCs effectively promoted the proliferation and activation of M2 macrophages expressing MMP13 and inhibited M1 macrophages to suppress the activation of HSCs, which together played synergistic roles in degrading liver fibrosis. Although research on the treatment of liver fibrosis by MSC transplantation has emerged in recent years, previous studies have usually focused on whether these stromal cells differentiate into hepatocyte-like cells to promote the regeneration of hepatic parenchymal cells and restore liver function [9, 35-37]. By contrast, the role of macrophages, as abundant innate immune cells in the liver, has been ignored. Our study is the first to demonstrate that the effects of BM-MSC transplantation on liver fibrosis are at least partially or even mainly based on their modulatory effect, especially via regulating macrophage subtypes. The change in macrophages plays a central role because it orchestrates cross-talk among different cell types, cytokines, and proteases to ultimately attenuate liver fibrosis.

\section{Abbreviations}

BM-MSCs: Bone marrow mesenchymal stromal cells; CCl4: Carbon tetrachloride; CD: Clusters of differentiation; DMEM: Dulbecco's modified Eagle media; GFP: Green fluorescent protein; HLA: Human leucocyte antigen; HSC: Hepatic stellate cell; IFN: Interferon; IL: Interleukin; i.p.: Intraperitoneal; MMP: Matrix metalloprotease; PBS: Phosphate buffer saline; SMA: Smooth muscle actin; TGF: Transforming growth factor; Th cell: Helper T cell; TNF: Tumor necrosis factor

\section{Acknowledgements}

The authors would like to thank Dr. Xiao-Kang Li and Dr. Liang Zhong for their technical assistance.

\section{Funding}

This work was funded by the National Natural Science Foundation of China (Grant No. 81500459).

\section{Availability of data and materials}

All data generated or analyzed during this study are included in this published article.

\section{Authors' contributions}

$X-Y L$ and $X-J M$ contributed to the conception and design, collection and assembly of data, data analysis and interpretation, and manuscript writing and editing. D-CC, WW, and LL contributed to the collection and/or assembly of data. KZ, MG, and PW contributed to the figure preparing. All authors read and approved the final manuscript.

\section{Ethics approval}

The experimental protocol was approved by the Committee on the Ethics of Animal Experiments of Shanghai Jiao Tong University. Animals were maintained at the same facility and all the animal experiments were conducted in the same facility in accordance with the experimental ethics committee.

\section{Consent for publication}

Not applicable.

\section{Competing interests}

The authors declare that they have no competing interests.

\section{Publisher's Note}

Springer Nature remains neutral with regard to jurisdictional claims in published maps and institutional affiliations.

\section{Author details \\ ${ }^{1}$ Department of Gastroenterology, Shanghai Ninth People's Hospital, Shanghai Jiao Tong University School of Medicine, Center for Specialty Strategy Research of Shanghai Jiao Tong University China Hospital Development Institute, No 639, Zhizaoju Road, Huangpu District, Shanghai 200011, China. ${ }^{2}$ Department of Pathology, Affiliated Obstetrics and Gynecology Hospital of Nanjing Medical University, Nanjing Maternity and Child Health Care Hospital, Nanjing, Jiangsu province, China. ${ }^{3}$ Department of Pathology, Traditional Chinese Medicine Hospital of Kunshan, Kunshan, Jiangsu province, China.}

Received: 28 June 2018 Revised: 11 December 2018 Accepted: 25 December 2018 Published online: 11 January 2019

References

1. Rockey DC, Bell PD, Hill JA. Fibrosis--a common pathway to organ injury and failure. N Engl J Med. 2015;372(12):1138-49.

2. Mokdad AA, Lopez AD, Shahraz S, Lozano R, Mokdad AH, Stanaway J, Murray CJ, Naghavi M. Liver cirrhosis mortality in 187 countries between 1980 and 2010: a systematic analysis. BMC Med. 2014;12:145.

3. Poynard T, Yuen MF, Ratziu V, Lai CL. Viral hepatitis C. Lancet. 2003; 362(9401):2095-100

4. Bataller R, Brenner DA. Liver fibrosis. J Clin Invest. 2005:115(2):209-18.

5. Jacobs SA, Roobrouck VD, Verfaillie CM, Van Gool SW. Immunological characteristics of human mesenchymal stem cells and multipotent adult progenitor cells. Immunol Cell Biol. 2013;91(1):32-9.

6. Gebler A, Zabel O, Seliger B. The immunomodulatory capacity of mesenchymal stem cells. Trends Mol Med. 2012;18(2):128-34.

7. Bartholomew A, Patil S, Mackay A, Nelson M, Buyaner D, Hardy W, Mosca J, Sturgeon C, Siatskas M, Mahmud N, et al. Baboon mesenchymal stem cells can be genetically modified to secrete human erythropoietin in vivo. Hum Gene Ther. 2001;12(12):1527-41.

8. Horwitz EM, Gordon PL, Koo WK, Marx JC, Neel MD, McNall RY, Muul L, Hofmann T. Isolated allogeneic bone marrow-derived mesenchymal cells engraft and stimulate growth in children with osteogenesis imperfecta: 
implications for cell therapy of bone. Proc Natl Acad Sci U S A. 2002;99(13): 8932-7.

9. Kuo TK, Hung SP, Chuang CH, Chen CT, Shih YRV, Fang SCY, Yang WW, Lee OK. Stem cell therapy for liver disease: parameters governing the success of using bone marrow mesenchymal stem cells. Gastroenterology. 2008;134(7): 2111-21.

10. Friedenstein AJ, Petrakova KV, Kurolesova Al, Frolova GP. Heterotopic of bone marrow. Analysis of precursor cells for osteogenic and hematopoietic tissues. Transplantation. 1968;6(2):230-47.

11. Sakaida I, Terai S, Yamamoto N, Aoyama K, Ishikawa T, Nishina H, Okita K. Transplantation of bone marrow cells reduces CCl4-induced liver fibrosis in mice. Hepatology. 2004;40(6):1304-11.

12. Eom YW, Kim G, Baik SK. Mesenchymal stem cell therapy for cirrhosis: present and future perspectives. World J Gastroenterol. 2015;21 (36):10253-61.

13. Rengasamy M, Singh G, Fakharuzi NA, Siddikuzzaman BS, Swamynathan $P$, Thej C, Sasidharan G, Gupta PK, Das AK, et al. Transplantation of human bone marrow mesenchymal stromal cells reduces liver fibrosis more effectively than Wharton's jelly mesenchymal stromal cells. Stem Cell Res Ther. 2017:8(1):143

14. Mantovani A, Sica A, Locati M. Macrophage polarization comes of age. Immunity. 2005:23(4):344-6.

15. Gordon S. Alternative activation of macrophages. Nat Rev Immunol. 2003; 3(1):23-35.

16. Pesce JT, Ramalingam TR, Mentink-Kane MM, Wilson MS, El Kasmi KC, Smith AM, Thompson RW, Cheever AW, Murray PJ, Wynn TA. Arginase-1expressing macrophages suppress Th2 cytokine-driven inflammation and fibrosis. PLoS Pathog. 2009;5(4):e1000371.

17. Herbert DR, Holscher C, Mohrs M, Arendse B, Schwegmann A, Radwanska M, Leeto M, Kirsch R, Hall P, Mossmann H, et al. Alternative macrophage activation is essential for survival during schistosomiasis and downmodulates Thelper 1 responses and immunopathology. Immunity. 2004;20(5):623-35.

18. Karlmark KR, Weiskirchen R, Zimmermann HW, Gasssler N, Ginhoux F, Weber C, Merad M, Luedde T, Trautwein C, Tacke F. Hepatic recruitment of the inflammatory $\mathrm{Gr} 1(+)$ monocyte subset upon liver injury promotes hepatic fibrosis. Hepatology. 2009:50(1):261-74.

19. Louka ML, Ramzy MM. Involvement of fibroblast-specific protein 1 (S100A4) and matrix metalloproteinase-13 (MMP-13) in CCl4-induced reversible liver fibrosis. Gene. 2016;579(1):29-33.

20. Bennett RG, Heimann DG, Singh S, Simpson RL, Tuma DJ. Relaxin decreases the severity of established hepatic fibrosis in mice. Liver Int. 2014;34(3):416-26.

21. Kim EJ, Cho HJ, Park D, Kim JY, Kim YB, Park TG, Shim CK, Oh YK. Antifibrotic effect of MMP13-encoding plasmid DNA delivered using polyethylenimine shielded with hyaluronic acid. Mol Ther. 2011;19(2):355-61.

22. Duarte S, Baber J, Fujii T, Coito AJ. Matrix metalloproteinases in liver injury, repair and fibrosis. Matrix Biol. 2015;44-46:147-56.

23. Issa R, Zhou X, Constandinou CM, Fallowfield J, Millward-Sadler H, Gaca MD, Sands E, Suliman I, Trim N, Knorr A, et al. Spontaneous recovery from micronodular cirrhosis: evidence for incomplete resolution associated with matrix cross-linking. Gastroenterology. 2004;126(7):1795-808.

24. Constandinou C, Henderson N, Iredale JP. Modeling liver fibrosis in rodents. Methods Mol Med. 2005;117:237-50.

25. Soleimani M, Nadri S. A protocol for isolation and culture of mesenchymal stem cells from mouse bone marrow. Nat Protoc. 2009;4(1):102-6.

26. Zhang BC, Li Z, Xu W, Xiang CH, Ma YF. Luteolin alleviates NLRP3 inflammasome activation and directs macrophage polarization in lipopolysaccharide-stimulated RAW264.7 cells. Am J Transl Res. 2018;10(1):265-73.

27. Manuelpillai U, Lourensz D, Vaghjiani V, Tchongue J, Lacey D, Tee JY, Murthi P, Chan J, Hodge A, Sievert W. Human amniotic epithelial cell transplantation induces markers of alternative macrophage activation and reduces established hepatic fibrosis. PLoS One. 2012;7(6):e38631.

28. Friedman SL. Hepatic stellate cells: protean, multifunctional, and enigmatic cells of the liver. Physiol Rev. 2008;88(1):125-72.

29. Reeves HL, Friedman SL. Activation of hepatic stellate cells--a key issue in liver fibrosis. Front Biosci. 2002;7:d808-26.

30. Subramanian S, Pallati PK, Sharma P, Agrawal DK, Nandipati KC. TREM-1 associated macrophage polarization plays a significant role in inducing insulin resistance in obese population. J Transl Med. 2017;15(1):85,

31. Luo XY, Takahara T, Kawai K, Fujino M, Sugiyama T, Tsuneyama K, Tsukada K, Nakae S, Zhong L, Li XK. IFN-gamma deficiency attenuates hepatic inflammation and fibrosis in a steatohepatitis model induced by a methionine- and choline-deficient high-fat diet. Am J Physiol Gastrointest Liver Physiol. 2013;305(12):G891-9.

32. Tomita K, Tamiya G, Ando S, Ohsumi K, Chiyo T, Mizutani A, Kitamura N, Toda K, Kaneko T, Horie Y, et al. Tumour necrosis factor alpha signalling through activation of Kupffer cells plays an essential role in liver fibrosis of non-alcoholic steatohepatitis in mice. Gut. 2006;55(3):415-24.

33. Moles A, Sanchez AM, Banks PS, Murphy LB, Luli S, Borthwick L, Fisher A, O'Reilly S, van Laar JM, White SA, et al. Inhibition of RelA-Ser536 phosphorylation by a competing peptide reduces mouse liver fibrosis without blocking the innate immune response. Hepatology. 2013;57(2):817-28.

34. Shah S, Ma Y, Scherzer R, Huhn G, French AL, Plankey M, Peters MG Grunfeld C, Tien PC. Association of HIV, hepatitis C virus and liver fibrosis severity with interleukin-6 and C-reactive protein levels. AIDS. 2015;29(11): 1325-33.

35. Lam SP, Luk JM, Man K, Ng KTP, Cheung CK, Rose-John S, Lo CM. Activation of interleukin-6-induced glycoprotein 130/signal transducer and activator of transcription 3 pathway in mesenchymal stem cells enhances hepatic differentiation, proliferation, and liver regeneration. Liver Transpl. 2010 16(10):1195-206.

36. Banas A, Teratani T, Yamamoto Y, Tokuhara M, Takeshita F, Quinn G, Okochi $\mathrm{H}$, Ochiya T. Adipose tissue-derived mesenchymal stem cells as a source of human hepatocytes. Hepatology. 2007;46(1):219-28.

37. Lee KD, Kuo TK, Whang-Peng J, Chung YF, Lin CT, Chou SH, Chen JR, Chen YP, Lee OK. In vitro hepatic differentiation of human mesenchymal stem cells. Hepatology. 2004;40(6):1275-84.

\section{Ready to submit your research? Choose BMC and benefit from:}

- fast, convenient online submission

- thorough peer review by experienced researchers in your field

- rapid publication on acceptance

- support for research data, including large and complex data types

- gold Open Access which fosters wider collaboration and increased citations

- maximum visibility for your research: over $100 \mathrm{M}$ website views per year

At BMC, research is always in progress.

Learn more biomedcentral.com/submissions 